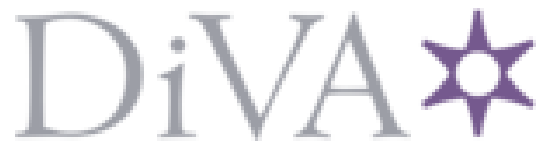

http://www.diva-portal.org

\title{
Postprint
}

This is the accepted version of a paper presented at 21st IEEE International Conference on Intelligent Transportation Systems (ITSC), NOV 04-O7, 2018, Maui, HI.

Citation for the original published paper:

Čičić, M., Johansson, K H. (2018)

Traffic regulation via individually controlled automated vehicles: a cell transmission model approach

In: 2018 21ST INTERNATIONAL CONFERENCE ON INTELLIGENT TRANSPORTATION SYSTEMS (ITSC) (pp. 766-771). IEEE

IEEE International Conference on Intelligent Transportation Systems-ITSC https://doi.org/10.1145/3178126.3178146

N.B. When citing this work, cite the original published paper.

Permanent link to this version:

http://urn.kb.se/resolve?urn=urn:nbn:se:kth:diva-244587 


\section{Traffic regulation via individually controlled automated vehicles: a cell transmission model approach}

\author{
Mladen Čičić \\ Department of Automatic Control \\ KTH Royal Institute of Technology \\ Stockholm, Sweden \\ cicic@kth.se
}

\author{
Karl Henrik Johansson \\ Department of Automatic Control \\ KTH Royal Institute of Technology \\ Stockholm, Sweden \\ kallej@kth.se
}

\begin{abstract}
The advent of automated, infrastructure-controlled vehicles offers new opportunities for traffic control. Even when the number of controlled vehicles is small, they can significantly affect the surrounding traffic. One way of regulating traffic is by using the automated vehicles as controlled moving bottlenecks. We present an extension of the cell transmission model that includes the influence of moving bottlenecks, consistently with the corresponding PDE traffic model. Based on this model, a control strategy is derived for traffic jam resolution. The strategy is tested in simulations, and shown to reduce the average travel time of surrounding vehicles, while also helping dissipate the traffic jam faster and ensuring the controlled vehicle avoids it.
\end{abstract}

\section{INTRODUCTION}

Traffic congestion has been a problem for a long time, and with increasing urbanization, its gravity can only be expected to increase in the future. The two major causes of traffic congestion are excess demand and poor traffic management [1]. Mitigating excess demand typically requires costly investment in transportation infrastructure, so it is desirable to attempt to reduce congestions by employing better traffic management.

The traditional methods of traffic control, such as variable speed limits [2], [3], ramp metering [4], [1] and their combinations [5], [6] have successfully been used to manage traffic systems, improve their efficiency and reduce congestions. While effective, these methods often require costly additional equipment for communicating or enforcing the control actions. Furthermore, the fact that this equipment is fixed at certain locations reduces the flexibility of these systems.

The increasing prevalence of intelligent vehicles and transportation systems, as well as development of a digital infrastructure that supports them, offers us new means of traffic management that have been underutilized so far. Even if the portion of intelligent vehicles is small, we can influence the surrounding traffic by using them as actuators, as was shown on the example of stop-and-go wave dissipation [7].

One way we can model actuation by an automated vehicle is by considering it a controlled moving bottleneck. If a vehicle moves slower than the surrounding traffic, it affects the traffic flow by limiting the number of vehicles that can move past it, as shown on Figure 1. Moving bottlenecks have mostly been considered in the literature in an experimental and empirical way [8], or in the framework of kinematic wave theory [9] and PDE traffic models [10], [11], [12]. While moving bottlenecks are usually seen as detrimental to traffic efficiency, the prospect of controlling them for traffic regulation has attracted some attention lately [13]. Heavyduty vehicles and vehicle platoons are typically slow-moving and naturally act as moving bottlenecks. In the future, we can expect fleet management systems to employ some centralized remote control over vehicles, using V2I communication to enable advanced route planning [14]. Since in addition, these vehicles would send their status to the fleet management system and receive reference speed profiles to follow, this makes them an ideal candidate for this purpose.

The problem we are focusing on in this paper is stopand-go wave dissipation, here assumed to be caused by a temporary reduction of road capacity. In contrast to some previous solutions that used variable speed limits (notable example being SPECIALIST [15]) or used autonomous vehicles in ring road traffic [7], we propose using a controlled automated vehicle acting as a moving bottleneck. In order to do this, we first model the influence of moving bottlenecks for the cell transmission model (CTM) in a way that is consistent with the corresponding PDE traffic model. Note that adopting a richer traffic model that captures the capacity drop phenomenon could broaden the range of applications of this method, but it would also prevent us from introducing moving bottlenecks in a concise and consistent manner, due to the lack of an equivalent first-order PDE model.

The main contribution of this paper is the introduction of moving bottlenecks to CTM, derived from PDE moving

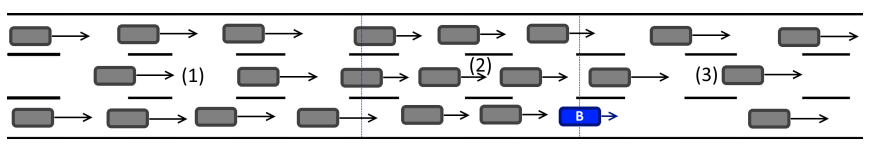

Fig. 1: Traffic separated into three zones caused by the moving bottleneck (vehicle indicated "B"): (1) unaffected oncoming vehicles, (2) congestion zone upstream, with increased traffic density and reduced speed and (3) "starvation" zone downstream, with lower traffic density and possibly higher speed. 
bottleneck models by solving a composite Riemann problem for cells adjacent to it. From the composite Riemann problem solution we obtain the traffic flow updates, as well as the position update for the controlled automated vehicle. This approach allows extensions to other PDE traffic models and different traffic phenomena, such as police cars. The resulting model enables control design using the speed of the moving bottleneck as control variable and cell traffic densities as measurements. In the simulation study, we present one control law example, which was shown to improve the efficiency of the traffic by faster dissipating the traffic jam and reducing average travel time.

The paper is organized as follows. In Section II we present the well-known CTM and Lighthill-WhithamRichards (LWR) PDE model. In Section III we introduce the moving bottleneck model, and show how to include it into the CTM. Next, in Section IV we give an example of traffic control using a controlled moving bottleneck. Finally, in Section $\mathrm{V}$ we demonstrate the use of the model through simulations and then conclude in Section VI.

\section{TRAFFIC MODEL}

Consider a stretch of highway with no on- or off-ramps. According to the standard CTM formulation [16], the evolution of traffic density $\rho_{i}$ in cell $i$ is given by

$$
\rho_{i}(t+1)=\rho_{i}^{t}+\frac{T}{L}\left(q_{i-1}(t)-q_{i}(t)\right), \quad i=1, \ldots N .
$$

Here we assume all cells are of same length $L$ and $T$ is the time step. By $q_{i}(t)$ we denote the outflow from cell $i$,

$$
q_{i}(t)=\min \left(V \rho_{i}(t), V \sigma, W\left(P-\rho_{i+1}(t)\right)\right),
$$

where $V$ is the free flow speed, $W$ the congestion wave speed, $\sigma$ the critical density, at which we have maximum flow and $P$ the jam density. In order to obtain a triangular fundamental diagram, we set the congestion wave speed to $W=V \frac{\sigma}{P-\sigma}$, so that $V \sigma=W(P-\sigma)$. We can handle the boundaries of the model by separately defining either the flow into the first cell $q_{0}(t)$ and out of the last cell $q_{N}(t)$, or boundary traffic densities $\rho_{0}(t)$ and $\rho_{N+1}(t)$.

This model was shown [17] to be equivalent to a discretization of the LWR model using the Godunov scheme. The LWR model is a non-linear first-order partial differential equation,

$$
\partial_{\tau} \rho+\partial_{x}(\rho \cdot v(\rho))=0,
$$

where $v(\rho)$ is the traffic speed expressed as a function of traffic density. To avoid confusion, we will use $\tau$ to denote the (continuous) time in PDEs and $t$ to denote the (discrete) time step in CTM. Using the piecewise-linear Newell-Daganzo flux function [16], we have

$$
Q(\rho)= \begin{cases}V \rho, & 0 \leq \rho \leq \sigma, \\ W(P-\rho), & \sigma<\rho \leq P,\end{cases}
$$

and the traffic speed dependence on traffic density is

$$
v(\rho)= \begin{cases}V, & 0 \leq \rho \leq \sigma, \\ W\left(\frac{P}{\rho}-1\right), & \sigma<\rho \leq P .\end{cases}
$$

Godunov discretization of (3) corresponds to taking piecewise constant initial conditions, $\rho(0, x)=\rho_{i}(t), x \in\left(X_{i}, X_{i+1}\right)$ and, with boundary conditions $\rho(0, x)=\rho_{0}(t), x<X_{1}, \quad$ and $\quad \rho(0, x)=\rho_{N+1}, x>X_{N}+L$, solving the composite Riemann problem for time up to $\tau=T$. We can then obtain the cell traffic density at the next time step $\rho(t+1)$ by averaging the solution of the PDE $\rho(T, x)$ over the length of each individual cell.

Since the flux function is piecewise linear, the solution of (3), $\rho(\tau, x)$, will be piecewise constant in $x$ for every $\tau$, and it can be calculated by solving Riemann problems for cell interfaces. The solution will consist of one or two wavefronts, depending on the density upstream of the cell interface, $\rho_{\text {- }}$ and downstream, $\rho_{+}$. In case we have a congestion upstream $\rho_{-}>\sigma$, and free flow downstream $\rho_{+} \leq \sigma$, the solution will be a rarefraction fan, with two wavefronts,

$$
\rho(\tau, x)= \begin{cases}\rho_{-}, & x<-W \tau \\ \sigma, & -W \tau<x<V \tau, \\ \rho_{+}, & x>V \tau .\end{cases}
$$

Otherwise, the solution will have one wavefront,

$$
\rho(\tau, x)= \begin{cases}\rho_{-}, & x<\Lambda\left(\rho_{-}, \rho_{+}\right) \tau, \\ \rho_{+}, & x>\Lambda\left(\rho_{-}, \rho_{+}\right) \tau,\end{cases}
$$

where $\Lambda\left(\rho_{-}, \rho_{+}\right)$is the Rankine-Hugoniot transition speed,

$$
\Lambda\left(\rho_{-}, \rho_{+}\right)=\frac{Q\left(\rho_{+}\right)-Q\left(\rho_{-}\right)}{\rho_{+}-\rho_{-}} .
$$

\section{MOVING BOTTLENECK MODEL}

In this section we introduce the moving bottleneck into the traffic model. We first describe the Riemann problems that arise from it and then apply a Godunov-like scheme to obtain traffic flow updates.

Assume we have a vehicle in traffic, at position $\chi_{b}(\tau)$ and moving at speed $u_{b}$ that is lower than the speed of the surrounding traffic $v\left(\rho\left(\tau, \chi_{b}(\tau)_{+}\right)\right)$. This vehicle acts as a moving bottleneck, restricting the amount of traffic flow that can pass by it, as shown in Figure 1. We model this effect by using a different flow model at the position of the vehicle.

Let the flux function at the moving bottleneck position, $Q_{b}(\rho)$, be of the same form as (4), with different parameters,

$$
Q_{b}(\rho)= \begin{cases}V_{b} \rho, & \rho \leq \sigma_{b}, \\ W_{b}\left(P_{b}-\rho\right), & \rho>\sigma_{b},\end{cases}
$$

where $W_{b}=W \frac{V_{b}}{V}$.

In order to model the capacity reduction in presence of a bottleneck, we introduce a new parameter $\beta \in[0,1]$ that describes the severity of the bottleneck. Density parameters $\sigma$ and $P$ are reduced to

$$
\begin{aligned}
& \sigma\left(x_{b}\right)=\sigma_{b}=\sigma(1-\beta), \\
& P\left(x_{b}\right)=P_{b}=P(1-\beta) .
\end{aligned}
$$

Since it depends on the behaviour of drivers, we would have to experimentally determine $\beta$, but in general, it can be taken to be close to the portion of the road that the moving bottleneck takes. For example, if one of two lanes is blocked, we can set $\beta=0.5$, or we might choose a somewhat higher value to capture additional "friction" effects.

Furthermore, we allow the free flow speed at the position of the bottleneck $V_{b}$ to differ from the free flow speed elsewhere, possibly even as a function of $u_{b}$. This enables us to 
model the overtaking behaviour in more detail, with $V_{b}>V$ indicating eagerness, and $V_{b}<V$ indicating reluctance to overtake. To ensure that the Rankine-Hugoniot condition can be satisfied at moving bottleneck interfaces, we also need $u_{b} \leq V_{b} \leq \frac{V-u_{b} \beta}{1-\beta}$. As with $\beta, V_{b}$ depends on driver behaviour, and would have to be experimentally determined. We make the standing assumption that $V_{b}$ is constant, $V_{b}>V$ and $u_{b} \leq \frac{V}{\beta}-\frac{V_{b}(1-\beta)}{\beta}$.

To model the influence the moving bottleneck has on the surrounding traffic, we solve two Riemann problems, one for its head and one for its tail. We denote the traffic density upstream of the bottleneck as $\rho_{-}$and downstream $\rho_{+}$, and the traffic density in the bottleneck zone as $\rho_{b}$.

Consider first the head of the bottleneck. The Riemann problem corresponding to it is

$$
\begin{gathered}
\partial_{\tau} \rho+\partial_{x}\left(Q_{+}\left(\rho, u_{b}, x, \tau\right)\right)=0, \\
Q_{+}\left(\rho, u_{b}, x, \tau\right)= \begin{cases}Q_{b}(\rho), & x<u_{b} \tau, \\
Q(\rho), & x>u_{b} \tau,\end{cases} \\
\rho(0, x)= \begin{cases}\rho_{b}, & x<0, \\
\rho_{+}, & x>0 .\end{cases}
\end{gathered}
$$

We control the movement of the bottleneck, so the transition speed between the zones where different models are valid has to be equal to its speed $u_{b}$. The Rankine-Hugoniot condition for the discontinuity, $u_{b}\left(\rho_{+}-\rho_{b}\right)=Q\left(\rho_{+}\right)-Q_{b}\left(\rho_{b}\right)$, can only hold for $\rho_{+}=r_{f}\left(\rho_{b}, u_{b}\right)$ or $\rho_{+}=r_{c}\left(\rho_{b}, u_{b}\right)$, where

$$
\begin{aligned}
& r_{f}\left(\rho_{b}, u_{b}\right)=\frac{Q_{b}\left(\rho_{b}\right)-u_{b} \rho_{b}}{V-u_{b}}, \\
& r_{c}\left(\rho_{b}, u_{b}\right)=\frac{W P-Q_{b}\left(\rho_{b}\right)+u_{b} \rho_{b}}{W+u_{b}} .
\end{aligned}
$$

Note that for $V_{b}=V, r_{f}\left(\rho_{b}, u_{b}\right)$ does not depend on $u_{b}$.

Otherwise, the solution to the Riemann problem will be a rarefraction fan with an additional zone of traffic density $r_{f}\left(\rho_{b}, u_{b+}\right)$, where $u_{b+}=\min \left(u_{b}, v\left(\rho_{+}\right)\right)$(corresponding to zone (3) in Figure 1),

$$
\rho(\tau, x)= \begin{cases}\rho_{b}, & x<u_{b+} \tau \\ r_{f}\left(\rho_{b}, u_{b+}\right), & u_{b+} \tau<x<\Lambda\left(r_{f}\left(\rho_{b}, u_{b+}\right), \rho_{+}\right) \tau, \\ \rho_{+}, & x>\Lambda\left(r_{f}\left(\rho_{b}, u_{b+}\right), \rho_{+}\right) \tau .\end{cases}
$$

Next, the Riemann problem corresponding to the tail of the bottleneck is

$$
\begin{gathered}
\partial_{\tau} \rho+\partial_{x}\left(Q_{-}(\rho, x, \tau)\right)=0, \\
Q_{-}\left(\rho, u_{b}, x, \tau\right)= \begin{cases}Q(\rho), & x<u_{b} \tau, \\
Q_{b}(\rho), & x>u_{b} \tau,\end{cases} \\
\rho(0, x)= \begin{cases}\rho_{-}, & x<0, \\
\rho_{b}, & x>0 .\end{cases}
\end{gathered}
$$

Rankine-Hugoniot condition, $u\left(\rho_{b}-\rho_{-}\right)=Q_{b}\left(\rho_{b}\right)-Q\left(\rho_{-}\right)$, can only hold for $\rho_{-}=r_{f}\left(\rho_{b}, u_{b}\right)$ or $\rho_{-}=r_{c}\left(\rho_{b}, u_{b}\right)$. Otherwise, the entropy solution is

$$
\rho(\tau, x)= \begin{cases}\rho_{-}, & x<\Lambda\left(\rho_{-}, r_{c}\left(\rho_{b}, u_{b}\right)\right), \\ r_{c}\left(\rho_{b}, u_{b}\right), & \Lambda\left(\rho_{-}, r_{c}\left(\rho_{b}, u_{b}\right)\right)<x<u_{b} \tau, \\ \rho_{b}, & x>u_{b} \tau,\end{cases}
$$

and the zone of traffic density $r_{c}\left(\rho_{b}, u_{b}\right)$ corresponds to zone (2) in Figure 1.
Finally, by assuming that the traffic moves past the bottleneck at maximum flow rate, we can calculate $\rho_{b}$ as

$$
\rho_{b}= \begin{cases}\frac{V-u_{b}}{V_{b}-u_{b}} \rho_{ \pm}, & \rho_{ \pm}<r_{f}\left(\sigma_{b}, u_{b}\right), \\ \sigma_{b}, & \rho_{ \pm} \in I_{f c}\left(\rho_{b}, u_{b}\right), \\ \frac{W_{b} P_{b}-W P+\left(W+u_{b}\right) \rho_{ \pm}}{W_{b}+u_{b}}, & \rho_{ \pm}>r_{c}\left(\sigma_{b}, u_{b}\right),\end{cases}
$$

where $I_{f c}\left(\rho_{b}, u_{b}\right)=\left[r_{f}\left(\rho_{b}, u_{b}\right), r_{c}\left(\rho_{b}, u_{b}\right)\right]$, and

$$
\rho_{ \pm}= \begin{cases}\rho_{+}, & \Lambda\left(\rho_{+}, \rho_{-}\right) \leq u_{b}, \\ \rho_{-}, & \Lambda\left(\rho_{+}, \rho_{-}\right)>u_{b} .\end{cases}
$$

Whenever $v\left(\rho_{+}\right)<u_{b}$, we use $u_{b+}$ instead of $u_{b}$.

We can now apply a similar Godunov-like scheme to calculate the effects of the moving bottleneck on traffic flows of adjacent cells. If $X_{i} \leq x_{b}(t)<X_{i}+L$, where $x_{b}(t)$ is the position of the moving bottleneck at time $t$, the moving bottleneck is in cell $i$ and $i_{b}(t)=i$. For compactness, we will omit writing the time step for all CTM-related variables wherever it is obvious. The resulting model will be the same as the already described standard CTM (1)-(2) for $i \neq i_{b}$, $i \neq i_{b}-1$. For these two cells, we will have

$$
\begin{aligned}
q_{i_{b}} & =\min \left(V \rho_{i_{b}}, V \sigma, W\left(P-\rho_{i_{b}+1}\right)\right)+\Delta q_{b}, \\
q_{i_{b}-1} & =\min \left(V \rho_{i_{b}-1}, V \sigma, W\left(P-\rho_{i_{b}}\right)\right)+\Delta q_{b-} .
\end{aligned}
$$

The behaviour of the moving bottleneck is described by two additional states, its position $x_{b}(t)$ and the traffic density directly upstream of it $\rho_{b-}(t)$. The second additional state is necessery in order to properly model the flow of traffic past the bottleneck [12], effectively splitting the cell $i$ into two parts. We will keep $\rho_{i}(t)$ as a state and instead, calculate the traffic density downstream of the bottleneck as

$$
\rho_{b+}=\frac{L \rho_{i_{b}}-\left(x_{b}-X_{i_{b}}\right) \rho_{b-}}{L-\left(x_{b}-X_{i_{b}}\right)} .
$$

We obtain $\Delta q_{b}(t)$ and $\Delta q_{b-}(t)$, as well as updates $x_{b}(t+1)$ and $\rho_{b-}(t+1)$ by solving the composite Riemann problem

$$
\begin{gathered}
\partial_{\tau} \rho+\partial_{x}\left(Q\left(\rho, u_{b}, x, \tau\right)\right)=0, \\
Q\left(\rho, u_{b}, x, \tau\right)= \begin{cases}Q(\rho), & x \neq \chi_{b}(\tau), \\
Q_{b}(\rho), & x=\chi_{b}(\tau),\end{cases} \\
\dot{\chi_{b}}(\tau)=\min \left(u_{b}, v\left(\rho\left(\tau, \chi_{b}(\tau)_{+}\right)\right),\right. \\
\rho(0, x)= \begin{cases}\rho_{i_{b}-1}, & x<X_{i_{b}}, \\
\rho_{b-}, & X_{i_{b}}<x<x_{b}, \\
\rho_{b+}, & x_{b}<x<X_{i_{b}+1}, \\
\rho_{i_{b}+1}, & x>X_{i_{b}+1},\end{cases} \\
\chi_{b}(0)=x_{b},
\end{gathered}
$$

for $\tau$ up to $T$. The solution is easily obtained through front tracking, and an example is shown in Figure 2.

Formally, we may write the solution at $\tau=T$ as

$$
\left[\begin{array}{c}
\rho(T, x) \\
\chi_{b}(T)
\end{array}\right]=\mathcal{P}_{T}\left(\rho_{i_{b}-1}, \rho_{i_{b}}, \rho_{i_{b}+1} ; x_{b}, u_{b}, \rho_{b-}\right),
$$

and obtain the changes in traffic flow as

$$
\begin{aligned}
\Delta q_{b} & =\frac{1}{T} \int_{X_{i_{b}+1}}^{\rho_{i_{b}+2}}(T, x) d x-\max \left(\Lambda\left(\underline{\rho}_{i_{b}}, \rho_{i_{b}+1}\right), 0\right)\left(\rho_{i_{b}+1}-\underline{\rho}_{i_{b}}\right), \\
\Delta q_{b-} & =\frac{1}{T} \int_{X_{i_{b}-1}}^{X_{i_{b}}}(T, x) d x-\min \left(\Lambda\left(\rho_{i_{b}-1}, \bar{\rho}_{i_{b}}\right), 0\right)\left(\rho_{i_{b}-1}-\bar{\rho}_{i_{b}}\right),
\end{aligned}
$$




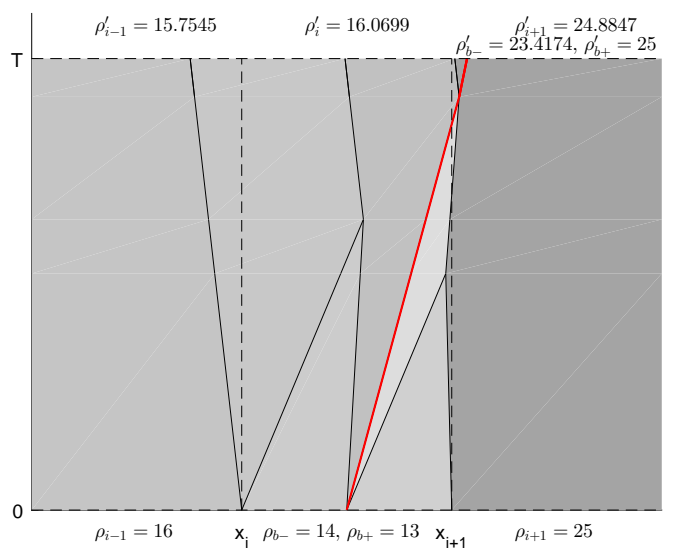

Fig. 2: Front tracking solution example for $\tau \in[0, T]$. Note that the moving bottleneck slows down when it enters the traffic from cell $i+1$.

where $\underline{\rho}_{i_{b}}=\min \left(\rho_{i_{b}}, \sigma\right)$ and $\bar{\rho}_{i_{b}}=\max \left(\rho_{i_{b}}, \sigma\right)$. We also obtain the new position of the bottleneck,

$$
x_{b}(t+1)=\chi_{b}(T),
$$

and the new traffic density upstream of it,

$$
\rho_{b-}(t+1)=\left\{\begin{array}{l}
\frac{\int_{X_{i_{b}(t+1)}}^{\chi_{b}(T)} \rho(T, x) d x}{\chi_{b}(T)-X_{i_{b}(t+1)}}, \rho_{i_{b}}(t+1) \in I_{f c}\left(\rho_{b}, u_{b}\right), \\
\rho_{i}(t+1), \quad \rho_{i_{b}}(t+1) \notin I_{f c}\left(\rho_{b}, u_{b}\right) .
\end{array}\right.
$$

To summarize, the model we propose is CTM (1)-(2), augmented by adding the effect of the moving bottleneck (7) onto the traffic flows from cells $i_{b}$ and $i_{b}-1$ (5). To properly model the dynamics of the moving bottleneck, we require adding two additional states (8) and (9) obtained from the solution (6) of the composite Riemann problem. The proposed model is simple and tractable, as well as consistent with the PDE moving bottleneck traffic models.

\section{TRAFFIC CONTROL VIA CONTROLLED MOVING BOTTLENECK}

In this section we derive a control law for moving traffic jam dissipation and avoidance, in accordance with the previously derived model. We are using the speed of controlled automated vehicle $u_{b}(t)$ as a control variable, and assuming that we know cell traffic densities $\rho_{i}(t)$. For readability, $(t)$ will be omitted.

We denote by $x_{d}$ and $x_{c}$ the positions of traffic jam head and tail, $x_{b}$ the position of the automated vehicle and $\rho$ the vector of cell traffic densities. The traffic jam encompasses a number of cells, $x_{c}=X_{i_{c}}, x_{d}(t)=X_{i_{d}}$, and we say that a cell $i$ is a part of the traffic jam if $\rho_{i}>\rho_{j}$ where $\rho_{j}>\sigma$ is some predefined density. The speed of the automated vehicle will be controlled as $u_{b}=\mathcal{U}\left(x_{b}, x_{c}, x_{d}, \rho\right)$, within some limits,

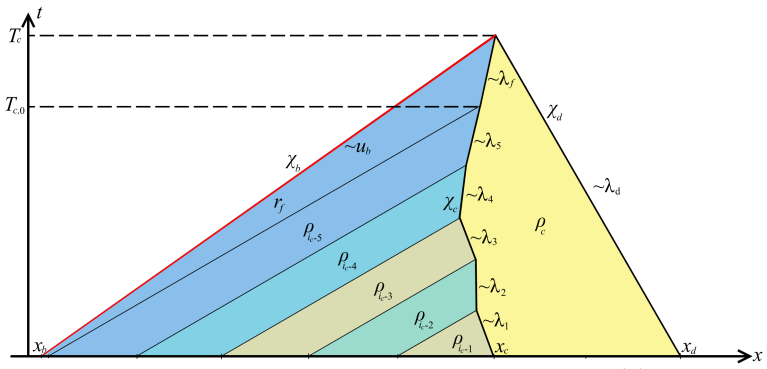

Fig. 3: Front tracking calculation of $u_{b}(t)$. $u_{b} \in\left[u_{\text {min }}, u_{\text {max }}\right]$. The control law $\mathcal{U}$ is a static mapping from its arguments to $u_{b}$. It is calculated and applied at every $t$, taking into account the updated states. The calculation of $\mathcal{U}$ will be described in the remainder of this section, and is illustrated in Figure 3. A representation of the control loop is shown in Figure 4.

The control objective we consider here is, from the perspective of the controlled vehicle, avoiding the traffic jam. If there is no traffic jam ahead of the controlled vehicle, it can continue driving at speed $u_{0}$. However, if a traffic jam does appear, the controlled vehicle can reduce its speed, in order to make it dissipate faster. We calculate and apply some constant controlled automated vehicle speed $u_{b}$ so that the congestion downstream of it is cleared as soon as possible and, if feasible, so that the vehicle avoids the congestion with minimum delay. Since $u_{b}>u_{\text {min }}$, this might not always be possible, in which case the vehicle will move at its minimum speed until it has passed the traffic jam.

For the vehicle to avoid the traffic jam with minimum delay, at some $\tau=T_{c}$, we need

$$
\chi_{b}\left(T_{c}\right)=\chi_{c}\left(T_{c}\right)=\chi_{d}\left(T_{c}\right) .
$$

Here we denote by $\chi_{*}$ the predicted evolution of $x_{*}$ in PDE framework. We assume that the congestion head will move at some constant speed $\lambda_{d}$,

$$
\chi_{d}(\tau)=x_{d}+\lambda_{d} \tau, \quad \chi_{d}(0)=x_{d} .
$$

While the reduction of capacity is still in effect, this speed will be $\lambda_{d}=0$, and afterwards, while the traffic jam is being discharged, $\lambda_{d}=-W$. For the position of the controlled vehicle, we have

$$
\chi_{b}(\tau)=x_{b}+u_{b} \tau, \quad \chi_{b}(0)=x_{b} .
$$

From (10), we can calculate the dependence of $T_{c}$ on $u_{b}$,

$$
T_{c}\left(u_{b}\right)=\frac{x_{d}-x_{b}}{u_{b}-\lambda_{d}},
$$

and for the position of the traffic jam tail at $\tau=T_{c}$ we write

$$
\chi_{c}\left(T_{c}\right)=x_{c}+\Delta \chi_{c}\left(\rho, u_{b}\right) .
$$

The dynamics of $\chi_{c}(\tau)$ are hard to describe in closed form, but we may calculate $\Delta \chi_{c}$ by the composite Riemann problem for initial conditions

$$
\rho(0, x)= \begin{cases}r_{f}\left(\sigma_{b}, u_{b}\right), & x \leq x_{b}, \\ \rho_{i}, & x \in\left[x_{i}, x_{i+1}\right), x_{c}>x>x_{b}, \\ \rho_{c}, & x>x_{c},\end{cases}
$$

by front tracking for $\tau \in\left[0, T_{c}\right]$. Note that here we model the influence of the moving bottleneck by formally taking the initial traffic density to be equal to $r_{f}\left(\sigma_{b}, u_{b}\right)$ everywhere upstream of it. Then, we can write

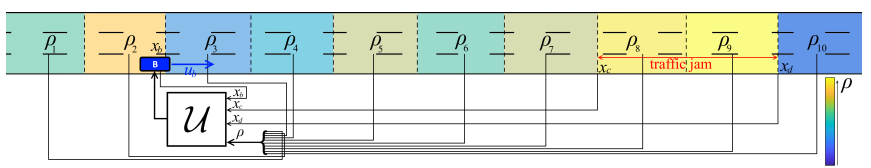

Fig. 4: Control loop example. Cell traffic densities $\rho_{i}$ are colorcoded (warmer is higher density). 


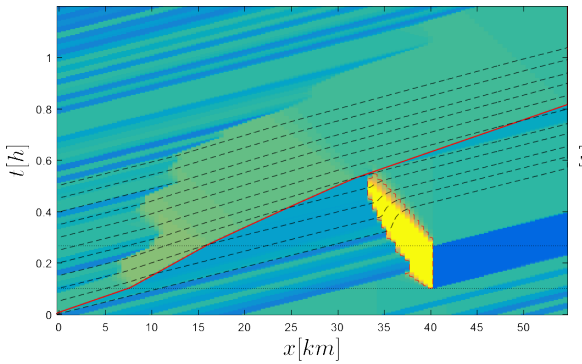

(a) Controlled

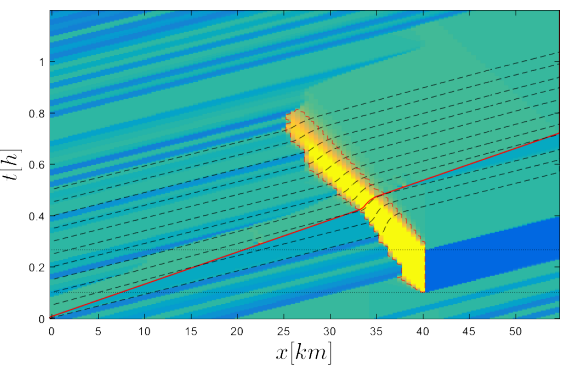

(b) Fast

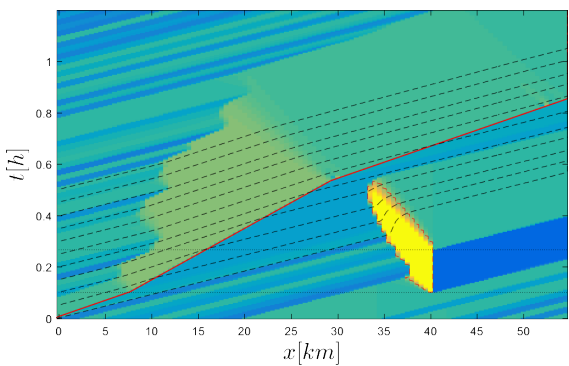

(c) Slow

Fig. 5: Traffic densities and moving bottleneck trajectories for the three cases.

$$
\begin{aligned}
\Delta \chi_{c}\left(\rho, u_{b}\right) & =\Delta \chi_{c, 0}(\rho)+\Delta \chi_{c, u_{b}}\left(u_{b}\right), \\
\Delta \chi_{c, 0}(\rho) & =\sum_{j=1}^{i_{c}-i_{b}} \lambda_{j} \Delta \tau_{j}, \\
\Delta \chi_{c, u_{b}}\left(u_{b}\right) & =\frac{x_{c}-x_{b}+\Delta \chi_{c, 0}(\rho)-T_{c, 0}(\rho) u_{b}}{u_{b}-\lambda_{f}\left(u_{b}\right)} \lambda_{f}\left(u_{b}\right),
\end{aligned}
$$

where

$$
\begin{aligned}
\lambda_{j} & =\Lambda\left(\rho_{i_{c}-j}, \rho_{c}\right), \\
\Delta \tau_{j} & = \begin{cases}\frac{L}{V-\lambda_{j}}, & i_{c}-j>i_{b}, \\
\frac{\left(i_{b}+1\right) L-x_{b}}{V-\lambda_{j}}, & i_{c}-j=i_{b},\end{cases} \\
T_{c, 0}(\rho) & =\sum_{j=1}^{i_{c}-i_{b}} \Delta \tau_{j}, \\
\lambda_{f}\left(u_{b}\right) & =\Lambda\left(r_{f}\left(\sigma_{b}, u_{b}\right), \rho_{c}\right) .
\end{aligned}
$$

Substituting (11), we calculate $u_{b}$ so that

$$
x_{c}+\Delta \chi_{c, 0}(\rho)+\Delta \chi_{c, u_{b}}\left(u_{b}\right)=x_{b}+\frac{u_{b}\left(x_{d}-x_{b}\right)}{u_{b}-\lambda_{d}} .
$$

at each $t$ until there is no more congestion ahead of the controlled vehicle. If $u_{b}$ is calculated to be less than $u_{\min }$ or greater than $u_{\max }$, we apply these extreme values instead.

\section{Simulation Results}

We tested the model and the control law in simulations. The simulation scenario in question is as follows:

1) $t<t_{0}$ : The traffic is in free flow, with heterogeneous traffic density. The controlled automated vehicle is moving at speed $u_{0}$.

2) $t_{0} \leq t<t_{1}$ : A traffic jam is caused by a reduction of capacity at position $x_{d}\left(t_{0}\right)$. The automated vehicle is acting as a moving bottleneck, and its speed is controlled so that the traffic jam is cleared as soon as possible.

3) $t \geq t_{1}, x_{b}(t) \leq x_{d}(t)$ : The reduction of capacity is removed and the traffic jam is being resolved. The automated vehicle's speed is controlled so that it dissipates and avoids the traffic jam with minimum delay.

4) $x_{b}(t)>x_{d}(t)$ : The vehicle has passed the traffic jam and it continues at speed $u_{0}$.

The simulation results for $u_{0}=80 \mathrm{~km} / \mathrm{h}$ are shown on Figure 5. Warmer colors represent higher traffic density and the traffic jam is outlined in dashed red line. The trajectory of the controlled automated vehicle is represented by full red line. The parameters of the fundamental diagram we used were $V=110 \mathrm{~km} / \mathrm{h}, \sigma=15 \mathrm{veh} / \mathrm{km}, P=70 \mathrm{veh} / \mathrm{km}$

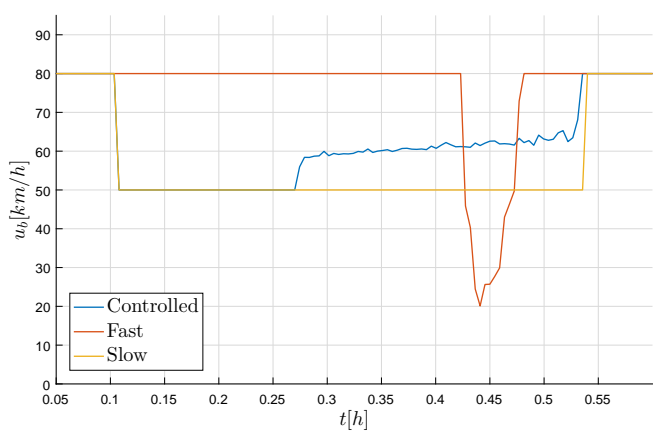

Fig. 6: Controlled vehicle speeds for the three cases.

and we take $\rho_{j}$ so that $v\left(\rho_{j}\right)=u_{0}$. Note that the traffic densities given here are per lane. The parameters of the moving bottleneck are $\beta=0.4$ and $V_{b}=120 \mathrm{~km} / \mathrm{h}$.

The reduction of capacity happens at $t_{0}=0.1$ and lasts for 15 minutes. During this time, the capacity at $x_{d}=40$ is reduced to $30 \%$. The minimum and maximum speeds of the controlled vehicle are taken to be $u_{\min }=50 \mathrm{~km} / \mathrm{h}$ and $u_{\max }=100 \mathrm{~km} / \mathrm{h}$. We compare three cases:

1) Controlled: The vehicle is controlled according to the control law from Section IV.

2) Fast: The vehicle does not reduce its speed, and continues at $u_{0}$.

3) Slow: The vehicle reduces its speed to $u_{\min }$ until there is no longer any traffic jam ahead of it.

We can see that by implementing such control strategy, the controlled vehicle avoids the traffic jam with little delay, while also helping resolve it faster. In second case, the controlled vehicle does traverse the road segment the fastest out of the three cases, but it does not help clear the traffic jam, and is forced to sharply reduce its speed while inside the congestion, as shown on Figure 6. In case the vehicle reduces its speed to $u_{\min }$, it helps resolve the traffic jam, but it is unnecessarily delayed.

We also tested the influence this control law has on the surrounding traffic, through 100 simulation runs for randomly generated background traffic and four different $u_{0}$. As performance metric, we will consider the average travel time (ATT) calculated for vehicles entering the road segment after the controlled automated vehicle. The ATTs are calculated for 51 vehicles travelling at average traffic speeds of the cells they are in. The first vehicle enters the road segment concurrently with the controlled vehicle, and the last one enters half an hour later. We also plot in dashed black line the trajectories of every fifth (to avoid cluttering the figures) such vehicle on 


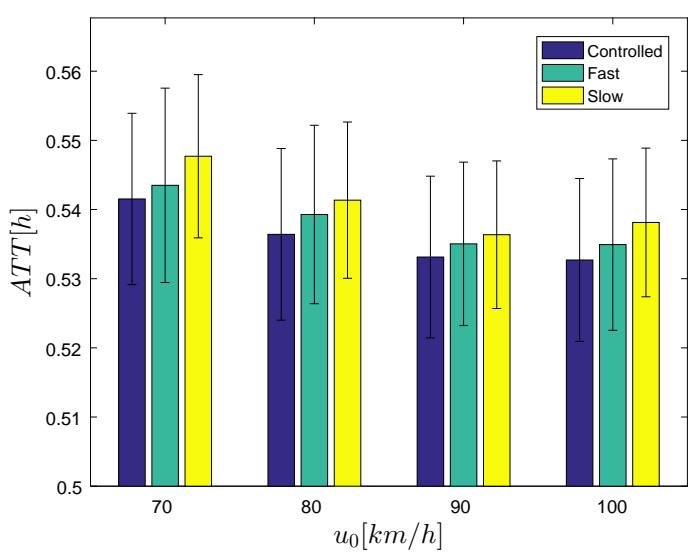

Fig. 7: Average travel times in hours comparison.

TABLE I: Average travel times. Without delays, $A T T_{0}=0.5 \mathrm{~h}$

\begin{tabular}{|r|c|c|c|}
\hline$u_{0}[\mathrm{~km} / \mathrm{h}]$ & Controlled & Fast & Slow \\
\hline 70 & 0.5415 & 0.5435 & 0.5477 \\
\hline 80 & 0.5364 & 0.5393 & 0.5414 \\
\hline 90 & 0.5331 & 0.5350 & 0.5364 \\
\hline 100 & 0.5327 & 0.5349 & 0.5381 \\
\hline
\end{tabular}

Figure 5. The results are shown on Figure 7 and in Table I.

We can see that employing the described control law leads to a reduction in ATT compared to both other cases, in addition to ensuring more desirable conditions for the controlled automated vehicle. In case the controlled vehicle keeps its speed at $u_{\min }$ until there is no more congestion ahead of it, the ATT is increased due to causing unnecessary additional congestion. Note that the control law was not explicitly derived in order to minimize ATT, so we might get even greater reduction by using optimization-based control.

\section{CONCLUSION}

In this paper we presented a simple way to include moving bottlenecks into the CTM framework that is consistent with the corresponding PDE model. The main purpose of such model is to facilitate deriving control laws traffic control problems through controlling a single automated vehicle that will act as a moving bottleneck. The example control problem we considered here is traffic jam dissipation with the added controlled-vehicle-centric control objective of minimizing controlled vehicle delay and avoiding the traffic jam, and the designed control law was shown to achieve good results. It was shown that we can reduce the average travel time by controlling a single automated vehicle.

We can envision many possible directions of future work. This approach can be extended to different, more complex traffic models, in particular the inverse-lambda phase transition CTM. It is also straightforward to extend this approach to consider different types of bottlenecks, such as police cars and long bottlenecks, as well as multiple bottlenecks. Finally, more complicated control problems can be tackled, for example including uncertain traffic information, multiple traffic jams or different constraints.

\section{ACKNOWLEDGEMENTS}

The research leading to these results has received funding from the European Union's Horizon 2020 research and inno- vation programme under the Marie Skłodowska-Curie grant agreement No 674875, VINNOVA within the FFI program under contract 2014-06200, the Swedish Research Council, the Swedish Foundation for Strategic Research and Knut and Alice Wallenberg Foundation. The first author is affiliated with the Wallenberg AI, Autonomous Systems and Software Program (WASP).

\section{REFERENCES}

[1] A. A. Kurzhanskiy and P. Varaiya, "Traffic management: An outlook," Economics of Transportation, vol. 4, no. 3, pp. 135 - 146, 2015.

[2] M. Papageorgiou, E. Kosmatopoulos, and I. Papamichail, "Effects of variable speed limits on motorway traffic flow," Transportation Research Record: Journal of the Transportation Research Board, no. 2047, pp. 37-48, 2008.

[3] M. Wang, W. Daamen, S. P. Hoogendoorn, and B. Van Arem, "Connected variable speed limits control and car-following control with vehicle-infrastructure communication to resolve stop-and-go waves," Journal of Intelligent Transportation Systems, vol. 20, no. 6, pp. 559572, 2016.

[4] M. Papageorgiou and A. Kotsialos, "Freeway ramp metering: An overview," in Intelligent Transportation Systems, 2000. Proceedings. 2000 IEEE, 2000, pp. 228-239.

[5] R. C. Carlson, I. Papamichail, M. Papageorgiou, and A. Messmer, "Optimal motorway traffic flow control involving variable speed limits and ramp metering," Transportation Science, vol. 44, no. 2, pp. 238$253,2010$.

[6] A. Hegyi, B. De Schutter, and H. Hellendoorn, "Model predictive control for optimal coordination of ramp metering and variable speed limits," Transportation Research Part C: Emerging Technologies, vol. 13, no. 3, pp. 185-209, 2005.

[7] R. E. Stern, S. Cui, M. L. D. Monache, R. Bhadani, M. Bunting, M. Churchill, N. Hamilton, R. Haulcy, H. Pohlmann, F. Wu, B. Piccoli, B. Seibold, J. Sprinkle, and D. B. Work, "Dissipation of stop-andgo waves via control of autonomous vehicles: Field experiments," Transportation Research Part C: Emerging Technologies, vol. 89, pp. $205-221,2018$.

[8] J. C. Muñoz and C. F. Daganzo, "Moving bottlenecks: a theory grounded on experimental observation," 15th International Symposium on Transportation and Traffic Theory, pp. 441-462, 2002.

[9] C. F. Daganzo and J. A. Laval, "On the numerical treatment of moving bottlenecks," Transportation Research Part B: Methodological, vol. 39, no. 1, pp. 31-46, 2005.

[10] J.-P. Lebacque, J. Lesort, and F. Giorgi, "Introducing buses into first-order macroscopic traffic flow models," Transportation Research Record: Journal of the Transportation Research Board, no. 1644, pp. 70-79, 1998.

[11] C. Lattanzio, A. Maurizi, and B. Piccoli, "Moving bottlenecks in car traffic flow: a pde-ode coupled model," SIAM Journal on Mathematical Analysis, vol. 43, no. 1, pp. 50-67, 2011.

[12] M. L. Delle Monache and P. Goatin, "A front tracking method for a strongly coupled pde-ode system with moving density constraints in traffic flow," Discrete and Continuous Dynamical Systems-Series S, vol. 7, no. 3, pp. 435-447, 2014.

[13] G. Piacentini, P. Goatin, and A. Ferrara, "Traffic control via moving bottleneck of coordinated vehicles," in 15th IFAC symposium on control in transportation systems, 2018.

[14] A. Alam, B. Besselink, V. Turri, J. Martensson, and K. H. Johansson, "Heavy-duty vehicle platooning for sustainable freight transportation: A cooperative method to enhance safety and efficiency," IEEE Control Systems, vol. 35, no. 6, pp. 34-56, 2015.

[15] A. Hegyi, S. Hoogendoorn, M. Schreuder, H. Stoelhorst, and F. Viti, "Specialist: A dynamic speed limit control algorithm based on shock wave theory," in Intelligent Transportation Systems, 2008. ITSC 2008. 11th International IEEE Conference on. IEEE, 2008, pp. 827-832.

[16] C. F. Daganzo, "The cell transmission model: A dynamic representation of highway traffic consistent with the hydrodynamic theory," Transportation Research Part B: Methodological, vol. 28, no. 4, pp. 269-287, 1994.

[17] J.-P. Lebacque, "The Godunov scheme and what it means for first order traffic flow models," Proceedings of the 13th International Symposium on Transportation and Traffic Theory, pp. 647-677, 1996. 